\section{Real-Time PCR: An Essential Guide}

\section{Kirsten Edwards, Julie Logan, and Nick Saunders}

\section{Horizon Bioscience, Norfolk, United Kingdom \\ ISBN: 0-954232-7-X}

Pages: 346, Price: £95; U.S. \$180

Real-time polymerase chain reaction (PCR) technique has advanced greatly over the past 10 years. This timely, comprehensive publication includes information on currently available instrumentation, fluorescent chemistries, assay design, optimization, and validation strategies. The background chapters are followed by chapters on quantification, single nucleotide polymorphism (SNP), mutation detection, and application of the various chemistries to clinical use in pathogen detection, gene expression, and human genetic testing. All of the chapters are well referenced; many of the contributing authors are recognized as respected experts in the field of real-time PCR.

Following a short overview of real-time PCR, the second chapter covers the various instruments currently on the market with a discussion on what features to look for when considering a purchase. The authors have put together a table of the machines, listing such details as the optics, the mode of detection (chargecoupled device camera or photomultiplier tube), the platform (96-well, glass capillary, etc.), and size and weight. The list contains every instrument except the latest offerings by ABI (7300 and 7500) and MJ (Chromo4). A couple of added features are given for some instruments but not others; for instance, the relative quantification software standard on the Stratagene Mx4000 and Mx3000p was not noted. Similar software is also available from $\mathrm{ABI}$ for their instruments. The list of websites after the references, containing general real-time PCR sites as well as those of the manufacturers and newsgroups, is an invaluable resource for both the novice and the veteran of real-time PCR.

Chapter 3 delves into the specific fluorescent chemistries, including intercalating dyes for generic detection of PCR product and templatespecific designs such as linear hydrolysis (Taqman) and hybridization probes, and conformational probes (i.e., Molecular Beacons, Scorpions). One design not included was the Lux primers, a trademark design from Invitrogen. There is ample discussion on how the various chemistries work, design parameters, and examples of specific applications. The publisher might consider some changes in the placement of the tables and figures in this chapter. Most of the tables and figures are placed at the front of the chapter but not referred to until much later in the text, making it awkward for the reader to refer to them. Some attention to the font size and type for Table 1, which is hard to read, and the gray scale in many other figures throughout the book would improve the depiction of the illustrations.

The next 3 chapters (4-6) cover assay set-up and optimization, assay validation, and the design and use of controls for quantification. Chapter 4 is a general overview; chapter 5 discusses the use of internal and external controls, with the emphasis on the design and optimization of a synthetic mimic as an internal control. Chapter 6 deals with developing and using a quantitative standard. All 3 chapters address the importance of assay optimization and how this relates to PCR efficiency. They also stress the use of appropriate controls to identify falsepositive results; more importantly, these chapters discuss how controls can identify false-negative results and their cause (PCR inhibitors, missing reagent components or test sample, or equipment problems), a must for diagnostic applications.

Chapter 7 deals with gene expression but I recommend that anyone considering real-time PCR read this chapter as a primer for what real-time PCR entails. The information on RNA extraction, reverse transcription, and amplification is extensive and includes discussion of the various enzymes, the master mixes and additives, and how they may or may not enhance recovery from any number of sources. The authors also cover optimization as it relates to reaction efficiency and relative versus absolute quantification. The monitoring of gene expression levels in response to viral load or cancer-producing tumor cells has become a critical part of treatment strategies, and the need for rapid, reliable assays has been effectively addressed with quantitative real-time PCR.

Comparison of how the different probe types (linear hybridization, hydrolysis, and conformational) and the Scorpion-labeled primer work in mutation detection is covered in chapters 8 and 9. Examples are given for how real-time PCR detection can be applied to identify human genetic diseases (Factor V Leiden and cystic fibrosis, for example) or to diagnose drug-resistant bacteria for proper drug therapy. Chapter 9 discusses the application of the ARMS (amplification refractory mutation system) technique for discrimination and selection of low levels of a mutant in a high background of wild-type DNA. Another unique real-time assay, nucleic acid sequence-based amplification (NASBA), is discussed in chapter 10. These isothermic NASBA assays are used most often for RNA detection and/or quantification. The reactions require avian myeloblastosis virus reverse transcriptase, ribonuclease $\mathrm{H}$, T7 RNA polymerase in the master mix, and a thermostatic fluorimeter; the probe of choice is the Molecular Beacon. 
The final 2 chapters cover a myriad of applications used in clinical microbiology and the diagnosis of infectious diseases. Even though presented as an overview, the $>100$ references in chapter 11 illustrate how vast and varied the application of real-time PCR, and the technological advances to support its use, have become in the past decade. This publication would be a good addition to any laboratory as an up-to-date resource for both the novice and the experienced researcher.

\section{Karen McCaustland*}

${ }^{*}$ Centers for Disease Control and Prevention, Atlanta, Georgia, USA

Address for correspondence: Karen McCaustland, Centers for Disease Control and Prevention, Biotechnology Core Facility, Mailstop G36, 1600 Clifton Rd, Atlanta, GA 30333; fax: 404-639-1331; email: kam1@ cdc.gov

\section{Textbook-Atlas of Intestinal Infection in AIDS}

\section{Daniele Dionisio}

Springer-Verlag, Italia, Milano, Italy ISBN: 88-470-0174-9

Pages: 489, Price: U.S. $\$ 149.00$

Gastrointestinal tract infections are prominent in patients with AIDS. Infections may be caused by a variety of bacterial, fungal, viral, protozoal, and helminthic pathogens, and affect persons in both developing and industrialized countries. The problems are especially acute in resource-limited countries where little or no access to highly active antiretroviral therapy exists; the impact of illnesses associated with HIV is most pronounced in these countries.
Daniele Dionisio, an authority in parasitology, has assembled a new treatise, Textbook-Atlas of Intestinal Infections in AIDS, that directly addresses this topic. In 489 pages, the work includes much background, including a fascinating chapter by Esther Diane on the history of the discovery of intestinal parasites. The work and illustrations of parasitic life cycles by Dionisio and colleagues are illuminating for all students of infectious diseases.

Much of the book addresses particular agents and the diseases they cause. A particular strength is the numerous illustrations. Although varying in quality, they represent an enormous compendium of information about these common problems. The figures on microscopic and ultrastructural pathology are particularly strong.

This volume should be considered as a background text for the pathologist, gastroenterologist, or infectious disease specialist who concentrates on HIV infections. The illustrations and references provide readers access to materials not easily obtained. The sections on clinical management of particular conditions are relatively sparse, and healthcare workers with patients with active problems should consult with a more comprehensive text. In total, this textbook-atlas is a useful addition in the battle against HIV infections and its complications. The editor and multinational group of authors are to be congratulated on their scholarly work.

\section{Martin J. Blaser,*}

*New York University School of Medicine, New York, New York, USA

Address for correspondence: Martin J. Blaser, New York University School of Medicine, Department of Medicine, 550 First Ave, OBVA606, New York, NY, USA; fax: 212-2633969; email: Martin.Blaser@med.nyu.edu

\section{Public Health Response to Biological and Chemical Weapons: WHO Guidance}

\section{World Health Organization}

\section{ISBN: 92-4-154615-8}

Pages: 357; Price: U.S. \$34.20

In this manual, the World Health Organization (WHO) updates its guidance for governments in preparing for a possible terrorist attack with biological or chemical weapons. The book has something for virtually everyone who may have an interest in this topic, from government officials to clinicians, including information about the history of biological and chemical warfare, applicable international treaties, procedures for requesting WHO technical consultation, fundamentals of public health emergency response, basics of infectious diseases, treatment of patients with specific infectious or toxic exposures, physical properties of various agents, the utility of reconnaissance satellites for detecting weapons development, the management of food and water safety programs, etc.

This ubiquity and ambitiousness underlie the manual's limitations and strengths. At times the guidance is so general that is almost an inventory of truisms (e.g., "If it is found that the [emergency] control measures are not effective, they must be changed or modified.”); elsewhere, the manual is a detailed resource. Its utility for different users will depend on their backgrounds and information needs. The core chapter, Public Health Preparedness and Response, may disappoint those seeking more than general principles. Yet these principles merit articulation.

Descriptions of the sarin attack in Tokyo in 1995 and the anthrax attacks 